\title{
Controlling a VTOL in 2-DOF Subspaces
}

\author{
Aydin Yesildirek and Bara Emran
}

\begin{abstract}
A switching controller for vertical take off and landing (VTOL) vehicle is presented. Since the entire system belongs to a class of underactuated systems, it is decomposed in to three fully-actuated subsystems. Then, a set of constraints on the rotor speeds are given to cancel out some nonlinearities in input, hence ensure to obtain an affine in control dynamics that enables us to derive local feedback linearizing controllers and together with some associated Lyapunov functions. Finally, a controller scheduler based on Lyapunov functions is invoked to select the subsystem to be controlled and achieve the desired tracking motion. An illustration is given to show the effectiveness of the controller.
\end{abstract}

Index Terms-Quadrotor, lyapunov function, underactuated systems, VTOL.

\section{INTRODUCTION}

We face underactuated systems in a wide range of industrial applications varying from aerospace to robotics, flexible to mobile platforms. They may be formed due to some number of reasons such as the lack of sufficient actuators that may exist because of some cost constraints, some physical difficulties or limitations, in some cases unexpected actuator failures. However, when a system is intentionally built to operate as an underactuated system, we assume that it is controllable in the region of intended operations. In other words, there exists a feasible operation in the configuration space that is achievable using the selected actuation mechanism. For instance, using four rotors, it is possible to fly a quadrotor for surveillance purposes.

VTOL motion may certainly be obtained by using four-rotor helicopters, quadrotors, which are studied extensively in literature for many applications due to their low-cost, maintainability, and maneuverability features. Quadrotors, having only four rotors, do not have enough control richness in their structure to provide unrestricted flight in full vector space. Hence, the quadrotors are not fully linearizable. In this paper, we will consider tracking problem of a desired linear motion in the Cartesian space together with the heading angle.

There exist several approaches to obtain a suitable controller for this class underactuated systems. First solutions have been shown on linearized dynamical models around some predefined flight conditions. Classical PID algorithms have been demonstrated successfully due to their simplicity and proven reliability in practice. Multi-loop PID architectures derived for a particular flight profile over a

Manuscript received November 18, 2013; revised March 20, 2014.

Aydin Yesildirek is with the Department of Electrical Engineering, American University of Sharjah, UAE.

Bara Emran is with the Mechatronics Graduate Program, American University of Sharjah, UAE (e-mail: Bara.Emran@yahoo.com). trimmed model have been shown to perform well. For instance, a PID controller is derived and compared to LQR and backstepping techniques in [1]. In [2] a gain scheduled PID technique is applied to quadrotor with some fault conditions. There is an example of a sliding mode controller for altitude control is shown [3]. Effectiveness of backstepping under disturbance for attitude control is shown in [4]. An $H_{\infty}$ control application is shown in [5], dynamic inversion based control scheme is given in [6]. In [7], an adaptive sliding mode and a feedback linearization methods are compared for the quadrotor platform. A nonlinear controller for a simplified model of quadrotor using a control Lyapunov function (clf) [8] has been shown in [9].

Our focus is to develop a novel structure to enable us to control the linear motion and the heading angle of quadrotor.

\section{BACKGROUND}

\section{A. Underactuated Dynamical Systems}

Rigid body dynamics may be derived using Euler-Lagrange method will have the following general equations of motion.

$$
M(q) \ddot{q}+V_{m}(q, \dot{q}) \dot{q}+G(q)+F(\dot{q})+\tau_{d}=\tau
$$

with $q$ configuration space, $M, V_{m}$ some matrices $G(q)$ and $F(\dot{q})$ some vectors. Bounded unknown disturbances and the control input torque are denoted by $\tau_{d}$ and $\tau$ respectively. Underactuated systems are defined when the system DOF is larger than the number of actuators. For such systems, one can partition the configuration space $x=\left[\begin{array}{ll}x_{a} & x_{u}\end{array}\right] \in \mathrm{R}^{n}$ and rewrite the (1) as

$$
\frac{d}{d t}\left[\begin{array}{l}
x_{a} \\
x_{u}
\end{array}\right]=\left[\begin{array}{l}
f_{a} \\
f_{u}
\end{array}\right]+\left[\begin{array}{l}
g_{a} \\
g_{u}
\end{array}\right] u
$$

with $x_{a} \in \Omega_{a}$ actuated and $x_{u} \in \Omega_{u}$ unactuated vector spaces, $u \in \mathrm{R}^{n_{a}}, f_{i}, g_{i}$ for $i=a, u$ smooth vector fields in appropriate dimensions.

\section{B. Partial Feedback Linearization}

Reference [10] has shown the existence of a collocated controller

$$
u=\left(g_{a}^{T} g_{a}\right)^{-1}\left[-f_{a}(x)+v\right]
$$

with $v$ auxiliary control input that partially feedback linearizes underactuated system in (2) as

$$
\dot{x}_{a}=v
$$




$$
\dot{x}_{u}=\alpha_{u}(x)+\beta_{u}(x) v
$$

with $\alpha_{u}, \beta_{u} \in \mathrm{R}^{n} u \quad$ uncontrolled internal dynamics nonlinearities. When a mechanism is intentionally built as an underactuated system, it is understood that there exists a sufficient controller within the region of operation.

To extend the similar discussion in to the uncollocated space let us repartition $x_{u}$ into $n_{a}$-DOF subsystems. For an $n$-DOF system, let $N=\left[\frac{n}{n_{a}}\right]$. Then, (5) may be generalized as

$$
\frac{d}{d t}\left[\begin{array}{c}
x_{1} \\
x_{2} \\
\vdots \\
x_{N}
\end{array}\right]=\left[\begin{array}{c}
f_{1} \\
f_{2} \\
\vdots \\
f_{N}
\end{array}\right]+\left[\begin{array}{c}
g_{1} \\
g_{2} \\
\vdots \\
g_{N}
\end{array}\right] u
$$

If all $g_{i} g_{i}^{T}(x)$ are full rank matrices almost everywhere for $i=1,2, \cdots N$ then there exists $N$ controllers

$$
u_{i}=g_{i}^{T}\left(g_{i} g_{i}^{T}\right)^{-1}(x)\left[-f_{i}(x)+v_{i}\right]
$$

that partially feedback linearizes (6) in to $N$ subsystems as

$$
\begin{aligned}
& \dot{x}_{i}=v_{i} \\
& \dot{x}_{j}=\alpha_{j}(x)+\beta_{j}(x) v_{i} \quad \forall j \neq i
\end{aligned}
$$

with $x_{i} \in \Omega_{i} \subset \mathrm{R}^{n_{a}}$ sub-vectors. For such systems, the $v_{i}$ can only be designed to control the actuated $i^{\text {th }}$ subsystem with $n_{a}$-DOF. Without losing generality we can assume $n$ is a multiple of $n_{a}$.

Assumption 1: Any one of the $N$ subsystem is controllable when the entire system is stable within the region of operation. Although it may seem to be strong assumption, it is expected for the systems where the actuation mechanism is designed to be less than full DOF. This is also applicable for VTOLs.

\section{Switching Lyapunov Functions}

Let us try to formulate a dynamic representation of class of nonlinear systems under $N$-switching inputs as follows

$$
\dot{x}(t)=f\left(x, u_{i}(x) ; t\right)=\zeta_{i}(x ; t)
$$

with $x \in \Omega \subset \mathrm{R}^{n}, f\left(x, u_{i} ; t\right)=\zeta_{i}$ Lipschitz continuous vector fields, $i \in \ell ;\{1, \cdots, N\}$. Without loosing generality $\zeta_{i}(0)=0$ for all $i \in \ell$.

Let us, now, define a piecewise constant switching signal $\sigma(t):[0, \infty) \rightarrow \ell$. Assume that there is a finite number of switching instances in any finite interval defined by a sequence $T_{S}=\left\{t_{0}, t_{1}, \cdots\right\}$.

Let us define Lyapunov-like function first.

Definition 1: A system has Lyapunov-like function $V$ in $\Omega_{x}$ if
1) There exists $\alpha_{2}>\alpha_{1}>0$ such that

$$
\alpha_{1}\|x\|^{2}<V<\alpha_{2}\|x\|^{2} \quad \forall x \in \Omega_{x}
$$

2) Its derivative is negative definite within the piece-wise continues time intervals $\left[t_{k}, t_{k+1}\right)$

$$
\dot{V}(x)<0 \quad \forall x \in \Omega_{x} \backslash\{x=0\} \quad \text { and } \quad \dot{V}(0)=0
$$

3) It forms a decreasing sequence at the switching instances of $T_{S}$

$$
V\left(x\left(t_{k+1}\right) ; t_{k+1}\right)<V\left(x\left(t_{k}\right) ; t_{k}\right) \quad \text { for } t_{k+1}>t_{k}
$$

Theorem 1 ([11]): Given the N-switched system (9), suppose each vector field $\zeta_{i}$ has an associated Lyapunov-like function $V_{i}$ in the region $\Omega_{i}$ with equilibrium point $\bar{x}=0$, let $\sigma(t)$ be a switching sequence such that $\sigma(t)$ can take on the value $i$ only if $x(t) \in \Omega_{i}$, and in addition

$$
V_{i}\left(x\left(t_{i, k}\right)\right) \leq V_{i}\left(x\left(t_{i, k-1}\right)\right)
$$

where $t_{i, k}$ denotes the $k$ th time that $\zeta_{i}$ is switched in. Then (9) is Lyapunov stable.

\section{PROBlEM DEFINITION}

A quadrotor as a VTOL device having with four rotors is shown in Fig. 1. Controlling the entire 6-DOF in VTOL with only four actuators is not possible.

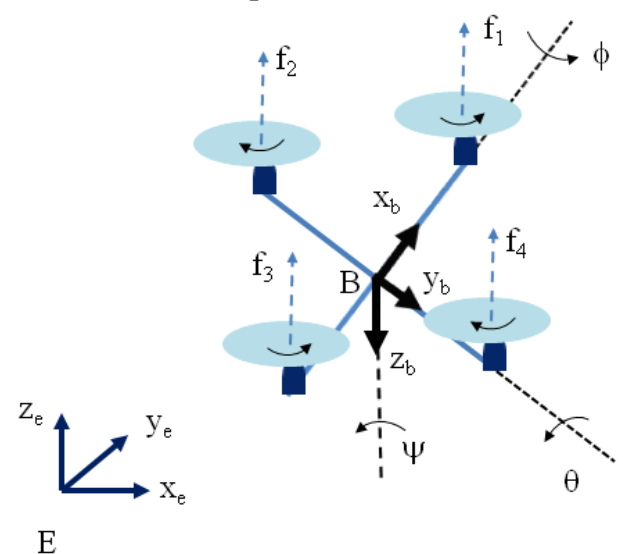

Fig. 1. Quadrotor frame.

Controlling the entire 6-DOF in VTOL with only four actuators is not possible. This structure is built in merely for the purpose of navigation in 3D Cartesian space with vertical takeoff and landing characteristics. Therefore, we consider that it is a functional underactuated system within the scope of this intended region of operation.

\section{A. VTOL Dynamics}

Relevant coordinate frames are shown in Fig. 1 as the earth-frame, $E$, is a fixed reference frame and the body frame, $B$, attached to the vehicle. An Euler-Lagrange based model is given in [6] as 


$$
\begin{gathered}
\ddot{x}=\frac{-\sin (\theta)}{m} u \\
\ddot{\theta}=\dot{\phi} \dot{\psi}\left(\frac{I_{z}-I_{x}}{I_{y}}\right)-\frac{J_{p}}{I_{y}} \dot{\phi} \Omega+\frac{l}{I_{y}} \tau_{\theta} \\
\ddot{y}=\frac{\cos (\theta) \sin (\phi)}{m} u \\
\ddot{\phi}=\dot{\theta} \dot{\psi}\left(\frac{I_{y}-I_{z}}{I_{x}}\right)-\frac{J_{p}}{I_{x}} \dot{\theta} \Omega+\frac{l}{I_{x}} \tau_{\phi} \\
\ddot{z}=-g+\frac{\cos (\theta) \cos (\phi)}{m} u \\
\ddot{\psi}=\dot{\phi} \dot{\theta}\left(\frac{I_{x}-I_{y}}{I_{z}}\right)+\frac{l}{I_{z}} \tau_{\psi}
\end{gathered}
$$

with inputs, lift force

$$
u=f_{1}+f_{2}+f_{3}+f_{4},
$$

and generalized torques

$$
\tau_{a}=\left[\begin{array}{c}
\tau_{\phi} \\
\tau_{\theta} \\
\tau_{\psi}
\end{array}\right]=\left[\begin{array}{c}
l\left(f_{3}-f_{1}\right) \\
l\left(f_{2}-f_{4}\right) \\
c\left(f_{1}-f_{2}+f_{3}-f_{4}\right)
\end{array}\right],
$$

The thrusts generated by rotor driven propellers may be shown as

$$
f_{i}=k \omega_{i}^{2}
$$

where $k$ is the lift constant, $\omega_{i}$ angular speed and the mismatch speed term

$$
\Omega=\omega_{2}+\omega_{4}-\omega_{1}-\omega_{3} .
$$

\section{B. Input Constraints}

The system dynamics (11)-(13) are not in the affine form. To transform the nonlinear control input terms, we constrain the angular velocities that generate lift force and generalized torques according to (14).

\begin{tabular}{ccccc}
\hline \hline & $w_{1}$ & $w_{2}$ & $w_{3}$ & $w_{4}$ \\
\hline pitch & $\omega_{0}$ & $\omega_{0}+\Delta$ & $\omega_{0}$ & $\omega_{0}-\Delta$ \\
roll & $\omega_{0}-\Delta$ & $\omega_{0}$ & $\omega_{0}+\Delta$ & $\omega_{0}$ \\
yaw & $\omega_{0}+\Delta$ & $\omega_{0}-\Delta$ & $\omega_{0}+\Delta$ & $\omega_{0}+\Delta$ \\
\hline \hline
\end{tabular}

Note that at the expense of reducing the effective control inputs to the already underactuated system we gain a set of equations of motion in the affine form. Let us define three subsystems to control 2-DOF motion using the nominal speed $\omega$ and the speed variation $\Delta$ needed to achieve the desired motion. Hence, the system model can be written in the form of (6) where

$$
\begin{aligned}
& x_{1}=\left[\begin{array}{llll}
x & \theta & \dot{x} & \dot{\theta}
\end{array}\right]^{T} \in \Omega_{p} \\
& x_{2}=\left[\begin{array}{llll}
y & \phi & \dot{y} & \dot{\phi}
\end{array}\right]^{T} \in \Omega_{r} \\
& x_{3}=\left[\begin{array}{llll}
z & \psi & \dot{z} & \dot{\psi}
\end{array}\right]^{T} \in \Omega_{y}
\end{aligned}
$$

$\Omega_{i} \subset \mathrm{R}^{4}$ for $i=p, r, y$ defining pitch, roll and yaw subsystems respectively.

Required forces needed for the lateral motion can be obtained by small rotations around $\theta$ and $\phi$ angles. Therefore, to operate the VTOL it is sufficient to keep the angles bounded by

$$
-\frac{\pi}{2}<\phi<\frac{\pi}{2}, \quad-\frac{\pi}{2}<\theta<\frac{\pi}{2}
$$

\section{Error System Dynamics in Fully-Actuated Subspaces}

Let there be a piecewise continuous desired motion defined by the vector $q_{d}=\left[\begin{array}{llll}x_{d} & y_{d} & z_{d} & \psi_{d}\end{array}\right]^{T}$. Due to the underactuation constraints we relax the roll and pitch motion for the tracking problem. Let us study the tracking error in each subsystem.

\section{1) Pitch subsystem}

Let the position tracking error in pitch subspace be defined as

$$
e_{p}=\left[\begin{array}{l}
e_{x} \\
e_{\theta}
\end{array}\right]=\left[\begin{array}{l}
x_{d}-x \\
\theta_{d}-\theta
\end{array}\right]
$$

Note that $\theta_{d}$ is a virtual desired signal utilized to provide the motion in $x$-dimension. Now, define a filtered tracking error as

$$
s_{p}=\dot{e}_{p}+\Lambda_{p} e_{p}
$$

with $\Lambda_{p}=\operatorname{diag}\left\{\lambda_{x}, \lambda_{\theta}\right\}>0$. Its dynamics can be expressed by taking derivative and using (11) and (14) as

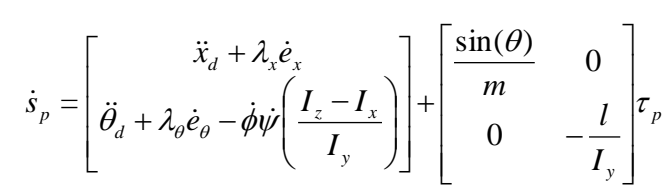

with $\tau_{p}=\left[\begin{array}{ll}u & \tau_{\theta}\end{array}\right]^{T}$.

\section{2) Roll subsystem}

The position tracking error in roll subspace may be defined as

$$
e_{r}=\left[\begin{array}{l}
e_{y} \\
e_{\phi}
\end{array}\right]=\left[\begin{array}{l}
y_{d}-y \\
\phi_{d}-\phi
\end{array}\right]
$$

Now, define filtered tracking errors

$$
s_{r}=\dot{e}_{r}+\Lambda_{r} e_{r}
$$

with $\Lambda_{r}=\operatorname{diag}\left\{\lambda_{y}, \lambda_{\phi}\right\}>0$. The filtered error dynamics can be expressed by taking derivative of (20) using (12) and (14) 


$$
\dot{s}_{r}=\left[\begin{array}{c}
\ddot{y}_{d}+\lambda_{y} \dot{e}_{y} \\
\ddot{\phi}_{d}+\lambda_{\phi} \dot{e}_{\phi}-\dot{\theta} \dot{\psi}\left(\frac{I_{y}-I_{z}}{I_{x}}\right)
\end{array}\right]-\left[\begin{array}{cc}
\frac{\cos (\theta) \sin (\phi)}{m} & 0 \\
0 & \frac{l}{I_{x}}
\end{array}\right]_{r}
$$

with $\tau_{r}=\left[u \tau_{\phi}\right]^{T}$.

\section{3) Yaw subspace}

Yaw subsystem is where the altitude and vertical takeoff and landing takes place. Therefore the desired position error vector is

$$
e_{y a w}=\left[\begin{array}{l}
e_{z} \\
e_{\psi}
\end{array}\right]=\left[\begin{array}{c}
z_{d}-z \\
\psi_{d}-\psi
\end{array}\right]
$$

Now, define the filtered tracking error vector

$$
s_{y}=\dot{e}_{y a w}+\Lambda_{y} e_{y a w}
$$

with $\Lambda_{y}=\operatorname{diag}\left\{\lambda_{z}, \lambda_{\psi}\right\}>0$. The filtered error dynamics can be expressed by taking derivative of (22), using (13) and the constraints of inputs as in (14)

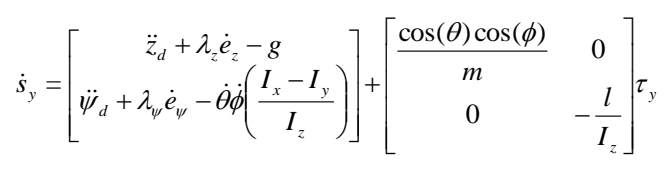

with $\tau_{y}=\left[\begin{array}{ll}u & \tau_{\psi}\end{array}\right]^{T}$.

\section{CONTROLler DEVElopMENT}

We will give subsystem controllers by the following Lemmas in the flight regimen. Then, define a control scheduler to select the desired fully-actuated subspace using Lyapunov functions.

\section{A. Fully-Actuated Controllers}

Desired flight regimen is achieved in one of the three subsystem operations, pitch, roll or yaw. For each subsystem, let's define three controllers in the structure of (7) by the following lemmas.

Theorem 2 (Pitch): Let controller for the pitch domain actuation be defined by the speeds

$$
\begin{aligned}
& \omega_{o}=\frac{1}{4 \sqrt{k}}\left(\sqrt{A_{p}}+\sqrt{B_{p}}\right) \\
& \Delta=\frac{1}{2 \sqrt{2 k}}\left(\sqrt{A_{p}}-\sqrt{B_{p}}\right)
\end{aligned}
$$

with

$$
\begin{aligned}
& A_{p}=u_{p}+\frac{\sqrt{2}}{l} \tau_{\theta} \\
& B_{p}=u_{p}-\frac{\sqrt{2}}{l} \tau_{\theta}
\end{aligned}
$$

and

$$
\begin{aligned}
& u_{p}=\frac{m}{\sin (\theta)}\left[\ddot{x}_{d}+\lambda_{x} \dot{e}_{x}+k_{v_{x}} s_{x}\right] \quad \theta \neq 0 \\
& \tau_{\theta}=\frac{I_{y}}{l}\left[\ddot{\theta}_{d}+\lambda_{\theta} \dot{e}_{\theta}-\dot{\phi} \dot{\psi}\left(\frac{I_{z}-I_{x}}{I_{y}}\right)+k_{v_{\theta}} s_{\theta}\right]
\end{aligned}
$$

with $k_{v_{y}}>0$ and with $k_{v_{\phi}}>0$. Then, the tracking error $e_{p}$ goes to zero exponentially in $\Omega_{p} \backslash\{\theta=0\}$.

Proof: Take a Lyapunov function

$$
V_{p}=\frac{1}{2} s_{p}^{T} P_{p} s_{p}>0
$$

with $s_{p}=\left[\begin{array}{ll}s_{x} & s_{\theta}\end{array}\right]^{T}$ and $P_{p}=P_{p}^{T}>0$. The error system dynamic given in (18) with the control (27) yields

$$
\dot{s}_{p}=-K_{v_{p}} s_{p}
$$

with

$$
K_{v_{p}}=\left[\begin{array}{cc}
k_{v_{x}} & 0 \\
0 & k_{v_{\theta}}
\end{array}\right]
$$

Thus, the time derivative of (28)

$$
\dot{V}_{p}=-s_{p} P_{p} K_{v_{p}} s_{p}=-\alpha_{p} \mathrm{P}_{s_{p}} \mathrm{P}^{2}<0
$$

Therefore $s_{p}$ goes to zero exponentially. Using the standard Lyapunov theorem one can conclude that $e$ goes to zero and using the Barbalat's extension so does $\dot{e}$. Hence the tracking is achieved in $\Omega_{p} \backslash\{\theta=0\}$.

Theorem 3 (Roll): Let the roll subspace dynamics be controlled by the speeds of

$$
\begin{aligned}
\omega_{o} & =\frac{1}{4 \sqrt{k}}\left(\sqrt{A_{r}}+\sqrt{B_{r}}\right) \\
\Delta & =\frac{1}{2 \sqrt{2 k}}\left(\sqrt{A_{r}}-\sqrt{B_{r}}\right)
\end{aligned}
$$

with

$$
\begin{aligned}
& A_{r}=u_{r}+\frac{\sqrt{2}}{l} \tau_{\phi} \\
& B_{r}=u_{r}-\frac{\sqrt{2}}{l} \tau_{\phi}
\end{aligned}
$$

with

$$
\begin{aligned}
& u_{r}=\frac{m}{\cos (\theta) \sin (\phi)}\left[\ddot{y}_{d}+\lambda_{y} \dot{e}_{y}+k_{v_{y}} s_{y}\right] \quad \phi \neq 0 \\
& \tau_{\phi}=\frac{I_{x}}{l}\left[\ddot{\phi}_{d}+\lambda_{\phi} \dot{e}_{\phi}-\dot{\theta} \dot{\psi}\left(\frac{I_{y}-I_{z}}{I_{x}}\right)+k_{v_{\phi}} s_{\phi}\right]
\end{aligned}
$$

with $k_{v_{y}}, k_{v_{\phi}}>0$. Then, $e_{2}$ goes to zero exponentially in $\Omega_{r} \backslash\{\phi=0\}$.

Proof: Take a Lyapunov function

$$
V_{r}=\frac{1}{2} s_{r}^{T} P_{r} s_{r}
$$

with $s_{r}=\left[\begin{array}{ll}s_{y} & s_{\phi}\end{array}\right]^{T}$ and $P_{r}=P_{r}^{T}>0$. Substituting the proposed controller (34) in to (21) results in 


$$
\dot{S}_{r}=-K_{v_{r}} s_{r}
$$

with $K_{v_{r}}$ positive diagonal matrix as in (30). Thus, the time derivative of (35)

$$
\dot{V}_{r}=-s_{r}^{T} P_{r} K_{v_{r}} s_{r}=-\alpha_{r}\left\|s_{r}\right\|^{2}<0
$$

Similar to pitch subspace, the exponentially convergence to zero tracking error is achieved in $\Omega_{r} \backslash\{\phi=0\}$.

Theorem 4 (Yaw): Finally, controller for the yaw subspace is constructed by

$$
\begin{aligned}
& \omega_{o}=\frac{1}{4 \sqrt{k}}\left(\sqrt{A_{y}}+\sqrt{B_{y}}\right) \\
& \Delta=\frac{1}{4 \sqrt{k}}\left(\sqrt{A_{y}}-\sqrt{B_{y}}\right)
\end{aligned}
$$

with

$$
\begin{aligned}
& A_{y}=u_{y}+\frac{1}{c} \tau_{\psi} \\
& B_{y}=u_{y}-\frac{1}{c} \tau_{\psi}
\end{aligned}
$$

with

$$
\begin{aligned}
& u_{y}=\frac{m}{\cos (\theta) \cos (\phi)}\left[\ddot{z}_{d}+\lambda_{z} \dot{e}_{z}-g+k_{v_{z}} s_{z}\right] \\
& \tau_{\psi}=\frac{I_{z}}{l}\left[\ddot{\psi}_{d}+\lambda_{\psi} \dot{e}_{\psi}-\dot{\phi} \dot{\theta}\left(\frac{I_{x}-I_{x}}{I_{z}}\right)+k_{v_{\psi}} s_{\psi}\right]
\end{aligned}
$$

with $k_{v_{z}}, k_{v_{\psi}}>0$. Then, $[z \psi \dot{z} \dot{\psi}]^{T}$ goes to $\left[z_{d} \psi_{d} \dot{z}_{d} \dot{\psi}_{d}\right]^{T}$ exponentially in $\Omega_{y}$.

Proof: Take a Lyapunov function

$$
V_{y}=\frac{1}{2} s_{y}^{T} P_{y} s_{y}>0
$$

with

$$
s_{y}=\left[\begin{array}{ll}
s_{z} & \gamma s_{\psi}
\end{array}\right]^{T}
$$

Substituting the proposed controller (40) in (24) and having the time derivative of (42) result in

$$
\dot{s}_{y}=-K_{v_{y}} s_{y}
$$

Thus, the time derivative of (41)

$$
\dot{V}_{y}=-s_{y} P_{y} K_{v_{y}} s_{y}=-\alpha_{y} P s_{y} P^{2}<0
$$

Once again, having $s_{y}$ go to zero exponentially that implies that the tracking objective is achieved everywhere in $\Omega_{y}$.

\section{B. Main Result}

So far, we have derived three fully-actuated controllers for 2-DOF subsystems in three flight conditions. We, now, show a scheduler to select the proper controller for the subsystem needing to be controlled.

Theorem 5: Let the pair $\left(\tau_{i}, V_{i}\right)$ with $i=p, r, y$ define the fully-actuated subsystem controllers and the corresponding clf. Then, the control scheduler using

$$
u=\left\{u_{i}: V_{i}=\max \left\{V_{p}, V_{r}, V_{y}\right\}\right\}
$$

guarantees the tracking of the desired quadrotor motion in the sense of Lyapunov.

Let us consider

$$
V_{\sigma}=\max \left\{V_{p}, V_{r}, V_{y}\right\}
$$

This is a bounded function, since

$$
a_{l}^{i}\|x\|<V_{i} \leq a_{u}^{i}\|x\|
$$

with $i=p, r, y$, then

$$
\underline{a}\|x\| \leq V_{\sigma} \leq \bar{a}\|x\|
$$

with

$$
\underline{a}=\max \left\{a_{l}^{i}\right\}, \quad \bar{a}=\min \left\{a_{l}^{i}\right\} \quad \forall i .
$$

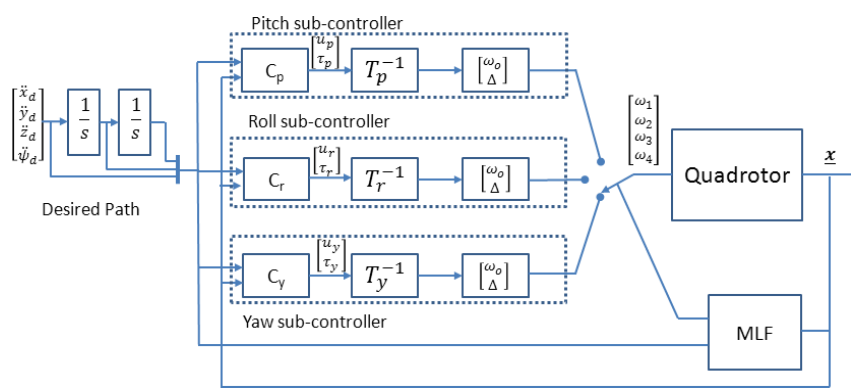

Fig. 2. Proposed control framework.

It is a piecewise decreasing function in the intervals defined by $\sigma(t)$. We have from Lemma 1-3 that at each interval, the selected control $u_{i}$ ensures the exponential decay of $V_{i}$.

Due to the continuity of $V_{i}$ for $i=p, r, y$, the function satisfies $V_{\sigma\left(t_{i+1}\right)}<V_{\sigma\left(t_{i}\right)}$ since $V_{i}$ 's cannot jump at the time of switching instants.

Therefore, $V_{\sigma}$ is a Lyapunov-like function. Invoking the Theorem 1 we conclude that the systemm is Lyapunov stable. This concludes the proof.

\section{ILLUSTRATIVE EXAMPLE}

To demonstrate the proposed controller we pick a small quadrotor with mass of $m=1 \mathrm{~kg}$, arm length $l=0.24 \mathrm{~m}$, moments of inertia about body frame axis $I_{x x}=I_{y y}=8.1 \cdot 10^{-3} \mathrm{kgm}^{2}, I_{z z}=14.2 \cdot 10^{-3} \mathrm{kgm}^{2}$, motor inertia $J=104 \cdot 10^{-8} \mathrm{kgm}^{2}$, thrust factor $k=54.2 \cdot 10^{-6} \mathrm{Ns}^{2}$, drag factor $d=1.1 \cdot 10^{-6} \mathrm{Nms}^{2}$. The limits on the motor actuators are set to be $500 \mathrm{rad} / \mathrm{s}$. 
TABLE I: THE DESIRED TRAJECTORY

\begin{tabular}{cc}
\hline \hline Time & Desired path \\
\hline$\left[0, T_{1}\right)$ & $\left(x_{o}, y_{o}, \frac{z_{1}}{T_{1}} t, 0\right)$ \\
{$\left[T_{1}, T_{2}\right)$} & $\left(x_{o}, y_{o}, z_{1}, \frac{\psi_{1}}{T_{2}-T_{1}}\left(t-T_{1}\right)\right.$ \\
{$\left[T_{2}, T_{3}\right)$} & $\left(x_{o}+R \sin \left(w\left(t-T_{2}\right)\right), y_{o}-R+R \cos \left(w\left(t-T_{2}\right)\right), z_{1}, \psi_{1}\right)$ \\
{$\left[T_{3}, T_{4}\right)$} & $\left(x_{o}+\frac{x_{1}-x_{o}}{T_{2}}\left(t-3 T_{2}\right), y_{o}+\frac{y_{1}-y_{o}}{T_{2}}\left(t-3 T_{2}\right), z_{1}, \psi_{1}\right)$ \\
\hline \hline
\end{tabular}

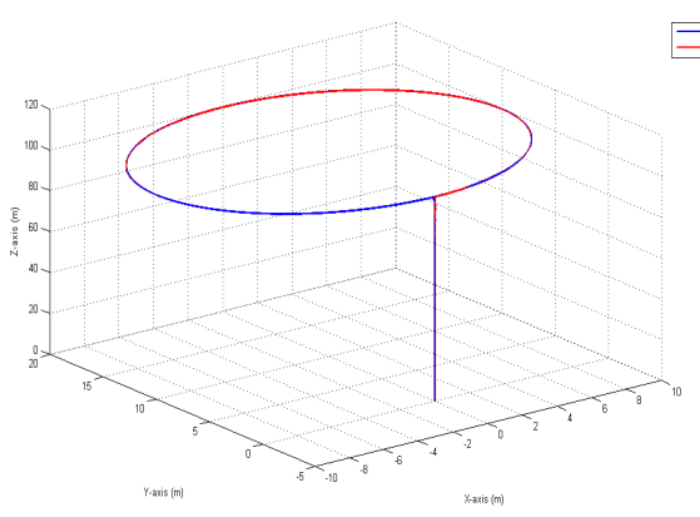

Fig. 3. Desired and actual paths.
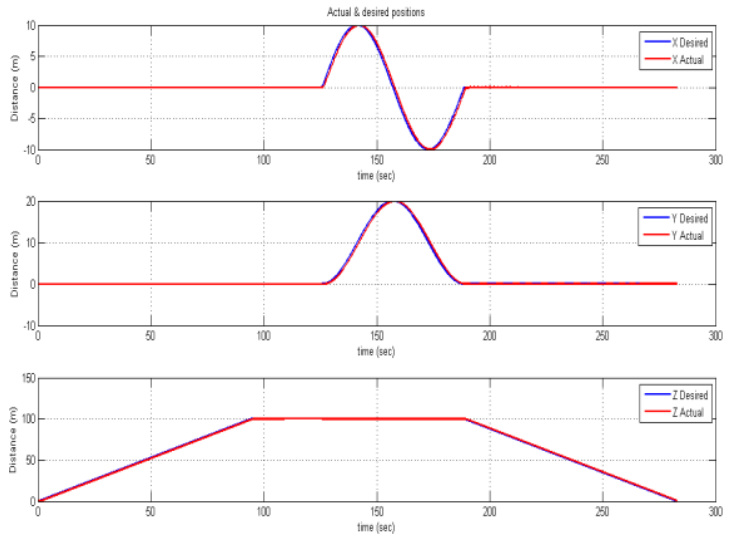

Fig. 4. Desired and actual positions.
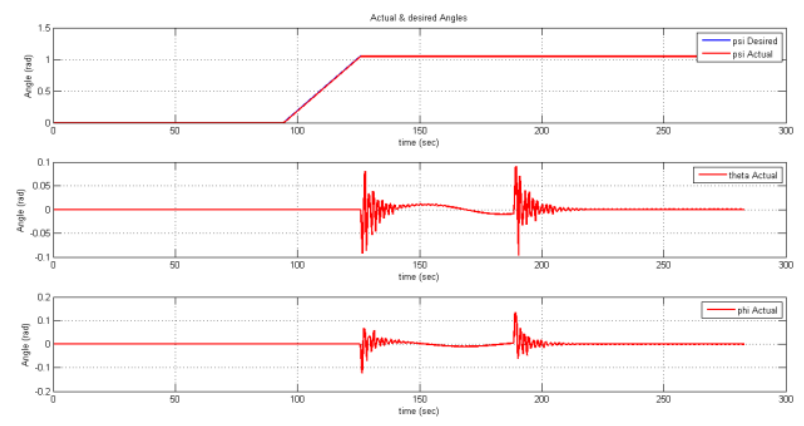

Fig. 5. Actual and desired angles.

The desired trajectory is defined by vertical takeoff, hover, circle and then vertical land by the trajectory as Control parameters and constructed by the sliding surface constants $\lambda_{i}=30$, control gain matrices $K_{v_{i}}=30 I$, Lyapunov function matrices $P_{i}=30 I$. Simulation results show the actual and desired 3D paths in Fig. 3. More detailed navigational errors are shown in Fig. 4 and Fig. 5.

\section{REFERENCES}

[1] S. Bouabdallah and R. Siegwart, "Full control of a quadrotor," in Proc. 2007 IEEE/RSJ International Conference on Intelligent Robots and Systems, Oct. 29- Nov. 2, 2007, no. 88, pp. 153-158.

[2] I. Sadeghzadeh, A. Mehta, A. Chamseddine, and Z. Youmin, "Active fault tolerant control of a quadrotor uav based on gainscheduled pid control," in Proc. 25th IEEE Canadian Conference on Electrical \& Computer Engineering, 2012, no. 88, pp. 1-4.

[3] R. Xu and U. Ozguner, "Sliding mode control of a quadrotor helicopter," in Proc. 45th IEEE Conference on Decision and Control, December 2006, no. 88, pp. 4957-4962.

[4] P. Castillo, P. Albertos, P. Garcia, and R. Lozano, "Simple real-time attitude stabilization of a quad-rotor aircraft with bounded signals," in Proc. 45th IEEE Conference on Decision \& Control, Dec. 13-15 2006, no. 88, pp. $1533-1538$.

[5] G. Raffo, M. Ortega, and F. Rubio, "Nonlinear h¥ controller for the quad-rotor helicopter with input coupling," in Proc. 18th IFAC World Congress, Aug. 28-Sep. 2, 2011, pp. $13834-13839$.

[6] A. Das, K. Subbarao, and F. Lewis, "Dynamic inversion with zerodynamics stabilisation for quadrotor control," IET Control Theory and Applications, vol. 3, no. 3, pp. 303-314, 2009.

[7] H. K. D. Lee and S. Sastry, "Feedback linearization vs. adaptive sliding mode control for a quadrotor helicopter," International Journal of Control, Automation, and Systems, vol. 7, no. 3, pp. 419-428, 2009.

[8] R. Freeman and P. Kokotovic, Robust Nonlinear Control Design, Statespace and Lyapunov Techniques, Boston, MA: Birkhauser, 2008.

[9] L. Sanchez, O. Santos, H. Romero, S. Salazar, and R. Lozano, "Nonlinear and optimal real-time control of a rotary-wing uav," in Proc. American Control Conference, June 27-29, 2012, no. 88, pp. $1533-1538$.

[10] M. Spong, "Underactuated mechanical systems," in Control Problems in Robotics and Automation, B. Siciliano and K. Valavanis, eds., London, UK: Springer-Verlag, 1997.

[11] M. Brainicky, "Studies in hybrid systems: Modeling, analysis, and control," Sc.D. dissertation, Electrical Engineering and Computer Science Dept., Massachusetts Institute of Technology, Cambridge, MA, June 2005.

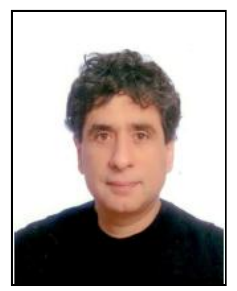

Aydin Yesildirek received his $\mathrm{PhD}$ in electrical engineering from the University of Texas at Arlington, TX in 1994, his MS degree is from Case Western Reserve University, OH, in 1992 and the B.S. degree in electronics and communication engineering from Istanbul Technical University in 1986. His research interests include autonomous vehicles, nonlinear control, intelligent and robust adaptive control systems. $\mathrm{He}$ is currently an associate professor in Electrical Eengineering Department and the director of the Mechatronics graduate program at American University of Sharjah, UAE.

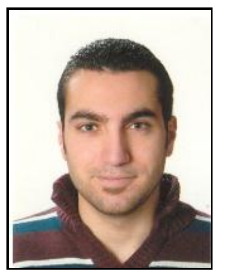

Bara Emran received his MS in mecahtonics program from American University of Sharjah, UAE in 2014. Also, He gained his BS in mechatronics engineering from the University of Jordan, Jordan in 2011.

Through his graduate study, he worked as a teacher and research assistant for four semesters. Also he worked as a lab adviser for two graduate level courses. During his work on variety type of robotic systems, he gained very good software skills in main engineering programs as well as advanced hardware skills.

Mr. Emran won several robotics contests. His graduation project was developing and implementing a laser guided autonomous vehicle which won the first place in the National Technology Parade in Jordan in 2011. His main areas of research interest are adaptive control, nonlinear control system, robotics, UAV and UGV. 